\title{
ROTATION INVARIANT COMPLEX EMPIRICAL MODE DECOMPOSITION
}

\author{
M Umair Bin Altaf ${ }^{1}$, Temujin Gautama ${ }^{2}$, Toshihisa Tanaka ${ }^{3}$, and Danilo P Mandic ${ }^{1}$ \\ ${ }^{1}$ Dept. Electrical and Electronic Engineering, Imperial College London, UK \\ \{muhammad.altaf,d.mandic \}@imperial.ac.uk \\ ${ }^{2}$ NXP Software, Philips Technology Campus Leuven, Belgium \\ temujin.gautama@philips.com \\ ${ }^{3}$ Dept. Electrical and Electronic Engineering, Tokyo Univ. of Agriculture and Technology, Japan \\ tanakat@cc.tuat.ac.jp
}

\begin{abstract}
A new method to extend the Empirical Mode Decomposition (EMD) into the complex domain is proposed. Unlike the existing method for EMD in the complex domain, this is achieved in a generic way so that the mathematical development of this method mirrors the algorithm defined for EMD in the real domain. The so derived Intrinsic Mode Functions (IMFs) are complex by design and are shown to provide a consistent framework for handling both real and complex data. The simulations on real world complex-valued signals illustrate the applications of the technique.
\end{abstract}

Index Terms-Signal Modality Characterization, Estimation, Signal resolution, Empirical Mode Decomposition

\section{INTRODUCTION}

Traditional time-frequency analysis methods are based on some sort of "a priori" mapping of a signal from the time domain into the frequency domain. These mappings are typically defined by 'basis functions' which are selected in order to ensure applicability to a wide class of signals. This poses problem for non-linear and nonstationary signals, which have time varying statistical characteristics and a single basis function fails as it cannot account for all variations in the signal. This compromises the physical significance of the analyzed output. The Fourier transform and Wavelet analysis belong to this category. The Fourier transform uses sinusoids of different frequencies as the basis function and is thus limited to analysis of linear and stationary data. The Wavelet analysis uses a set of finite support functions. The basis function are predefined and chosen to fit the type of data to be analyzed but even then it is not particularly useful for non-linear data [1]. There have been several attempts to define an adaptive basis which reflects the properties of data. One such technique is the Principle Component Analysis (or Karhunen-Loeve transform). It offers an adaptive basis which is defined a posteriori from the data but the assumptions inherent in this technique about the orthogonality of signal and interference might not be applicable to most signals.

Empirical Mode Decomposition (EMD) has been introduced by Huang et al. [1] and has been successfully used in many applications in various disciplines ranging from metrology to image analysis (for example see [2], [3] and [4]). It is a versatile data-driven signal analysis tool which provides new insights into the analysis of nonlinear and non-stationary data. Unlike conventional data analysis techniques, EMD imposes no prior assumptions on the data in the form of predefined basis functions and thus imposes no restriction on the analyzed signals. EMD operates in time-domain and adaptively decomposes a signal into a set of basis functions called the 'Intrinsic Mode Functions'(IMFs); the data can be considered to be mapped onto a space spanned by the IMFs. The Hilbert Transform of the IMFs introduces the notion of 'instantaneous frequency'. The combined application of EMD and Hilbert transform is known as the Hilbert-Huang Transform (HHT) [1].

Several important signal processing areas use complex-valued data structures, for which EMD in its current form is not designed, being limited to the analysis of real-valued data only. Thus, it is essential to develop an extension of EMD which suits complex-valued data. One such approach was proposed in [5], where Tanaka and Mandic have discussed the problem of extending EMD into the complex domain and they have developed an algorithm which is based on the inherent relationship between the positive and negative frequency components of a complex signal. The algorithm is relatively straight forward and has a rigorous mathematical background. The algorithm treats the positive and negative frequency component of the signal as two separate, independent signals. The EMD of these two signals results in two sets of IMFs: one for the positive frequency component of the signal and the other for the negative frequency part of the signal. The positive and negative frequency components are generally mutually dependent which leads to a loss of information in the signal. Although, this method preserves the dyadic filter bank property of EMD [6], it cannot be extended to higher-dimensional cases. Moreover, despite its successful operation in $\mathbb{C}$ and strict derivation, it is not generic and as such not a natural extension of the original real-valued EMD algorithm. The division of the signal into positive and negative frequency components also creates an ambiguity at the zero frequency.

To that cause, we propose a new way to decompose a complex signal with EMD by operating completely in $\mathbb{C}$. This is achieved by making use of complex splines which makes it possible to carry out all the arithmetic and algebraic operations of the algorithm in $\mathbb{C}$. This also provides a single set of IMFs, similar to the IMFs in the real EMD, but as desired the IMFs $\in \mathbb{C}$. The operation of the proposed complex EMD is demonstrated on modality characterization of real world complex-valued data.

\section{EMPIRICAL MODE DECOMPOSITION AND THE HILBERT TRANSFORM}

The empirical mode decomposition (EMD) aims at representing an arbitrary signal via a number of intrinsic mode functions (IMFs) $d_{k}[n]$ and the residual $r[n]$. By design, an IMF is a function for 
which the number of extrema and the number of zero crossings are either equal or they differ at most by one, together with the mean value of two envelopes associated with the local maxima and minima being zero. More precisely, for a real-valued signal $x[n]$, the EMD performs the mapping

$$
x[n]=\sum_{k=1}^{K} d_{k}[n]+r[n],
$$

where $\left\{d_{k}[n]\right\}_{k=1}^{K}$ is a set of IMFs and $r[n]$ is the residual. The first IMF can be obtained as follows [1]:

1. let $\tilde{x}[n]:=x[n]$,

2. identify all local minima and maxima of $\tilde{x}[n]$,

3. find an "envelope," $e_{\min }[n]$ (resp. $e_{\max }[n]$ ) which interpolates all local minima (resp. maxima),

4. extract the "detail," $d[n]=x[n]-\frac{1}{2}\left(e_{\min }[n]+e_{\max }[n]\right)$,

5. let $\tilde{x}[n]:=d[n]$ and go to step 2, repeat until $d[n]$ becomes an IMF.

Once the first IMF is obtained, to obtain the next IMF, the above procedure is applied to the residual $r[n]=x[n]-d[n]$. In the same spirit, by applying this procedure recursively, the remaining IMFs are calculated. The set of so obtained IMFs, in fact, represents a unique "time-frequency" analyzer which allows for the analysis of the instantaneous frequency, defined via the Hilbert transform [7].

For illustration, consider a continuous time real-valued signal $x(t)$ and apply the Hilbert transform to generate the corresponding analytic signal (for more information see [1])

$$
z(t)=\alpha(t)+j \beta(t)=A(t) e^{j \phi(t)},
$$

where $\{\alpha(t), \beta(t)\}$ is the Hilbert transform pair, $A(t)$ is the amplitude of $z(t)$, and $\phi(t)$ is its phase given by $\phi(t)=\arctan \left(\frac{\beta(t)}{\alpha(t)}\right)$. The instantaneous frequency $\omega(t)$ is derived from $\phi(t)$ as [1]

$$
\omega(t)=\frac{d \phi(t)}{d t} .
$$

It is this combination of the concept of instantaneous frequency and EMD that makes the EMD framework so powerful for time-frequency signal analysis. To illustrate this, further consider signal $x(t)$, which by means of EMD is described as $x(t)=r(t)+\sum_{k=1}^{K} d_{k}(t)$, where $\left\{d_{k}(t)\right\}_{k=1}^{K}$ is the set of IMFs representing "modes" and $r(t)$ is a residual of the decomposition. This way, we obtain a "spectgram" [1], given by $\left(t, \frac{d\left(d_{k}(t)\right)}{d t},\left|d_{k}(t)\right|\right)$, which helps us comprehend the time-frequency characteristics of a signal.

\section{EMPIRICAL MODE DECOMPOSITION FOR COMPLEX-VALUED DATA}

This technique, suggested in [5], is based on preserving the stochastic behaviour of EMD as a filter bank. Let $x[n] \in \mathbb{C}$ and $X\left(e^{j \omega}\right)$ be its discrete time Fourier transform. Now if $x[n]$ is analytic then the real and imaginary part of $x[n]$ are related by the Hilbert transform [7] and $X\left(e^{j \omega}\right)=0, \forall \omega<0$. Thus we can use the real part of the analytic signal in EMD and since EMD is complete [1], we can apply Hilbert transform the IMFs to get analytic IMFs. This would allow us to get around the problem of performing the EMD in complex domain. But $x[n]$ is generally not analytic so we have to convert these signals into analytic signals. This can be achieved with a filter which extracts the positive and negative frequencies of a signal and then time reverse the negative frequency components to get two analytic signals. Using such a filter, we obtain the positive and negative frequency parts of $X\left(e^{j \omega}\right)$ as $X_{+}\left(e^{j \omega}\right)$ and $X_{-}\left(e^{j \omega}\right)$ respectively. The Inverse Fourier transform of these signals provides the time domain complex signals $x_{c+}[n]$ and $x_{c-}[n]$. These time domain signals are analytic by construction and their imaginary parts can be discarded to yield the two real signals $x_{+}[n]$ and $x_{-}[n] . x_{+}[n]$ is the part of $x[n]$ corresponding to its positive frequency and $x_{-}[n]$ is its negative frequency part. Now, EMD can be applied on these real signals to obtain the IMFs.

$$
x_{+}[n]=\sum_{i=1}^{N^{+}} x_{i}[n]+r_{+}[n], x_{-}[n]=\sum_{i=-1}^{N^{-}} x_{i}[n]+r_{-}[n],
$$

Where $x_{i}[n]$ and $r_{+}[n]$ for $i>0$ are the IMFs and residuals, respectively, for $x_{+}[n]$ and $x_{i}[n]$ and $r_{-}[n]$ for $i<0$ are the IMFs and residuals, respectively, for $x_{-}[n] . N^{+}$and $N^{-}$are the number of IMF's for the positive and negative frequency part respectively. The signal $x[n]$ can be regenerated as follows:

$$
x[n]=\sum_{i=-N^{-}, i \neq 0}^{N^{+}} x_{i}[n]+r[n],
$$

The $i-t h$ complex IMF $c_{i}[n]$ is given as:

$$
c_{i}[n]= \begin{cases}x_{i}[n]+j \mathscr{H}\left[x_{i}[n]\right], & i=1, \ldots, N_{+}, \\ \left(x_{i}[n]+j \mathscr{H}\left[x_{i}[n]\right]\right)^{*}, & i=-N_{-}, \ldots,-1 .\end{cases}
$$

Where $(.)^{*}$ is the complex conjugate operator and $\mathscr{H}[$.$] denotes the$ Hilbert transform. Equations (6) and (7) define the complex EMD of $x[n]$.

Figures 2 show the IMFs calculated for a complex signal shown in Figure 1 with this method. This signal is the real world measurement of wind speed, $v(t)$ and wind direction, $\phi(t)$, which can be represented as a single complex variable, $x(t)=v(t) e^{j \phi(t)}[8]$. As can be seen in Figure 2, there are problems with this approach. Firstly, the EMD of a complex-valued signal obtained with this method has different number of IMFs for the positive and negative frequency part and as a result the analysis for the positive and negative frequencies would be not be aligned. This also prevents physically meaningful interpretation of the instantaneous frequency so generated for the two sets of IMFs. In addition, this method cannot be extended to higher dimensional cases due to the inherent limitations in representing a complex signal with its positive and negative frequency components.

\section{THE PROPOSED ROTATIONAL INVARIANT COMPLEX EMD}

A natural and generic way to extend EMD to the complex domain would be to operate completely in $\mathbb{C}$. This implies that all the basic steps for EMD described in Section 2: identification of extrema, interpolation between the extrema and other arithmetic operations will be carried out directly in the complex domain.

The first issue that needs to be addressed when extending the EMD to the complex domain with this method is the definition of an extremum, and a method for determining it. Indeed, in the complex domain, an extremum can be interpreted in different ways, for 


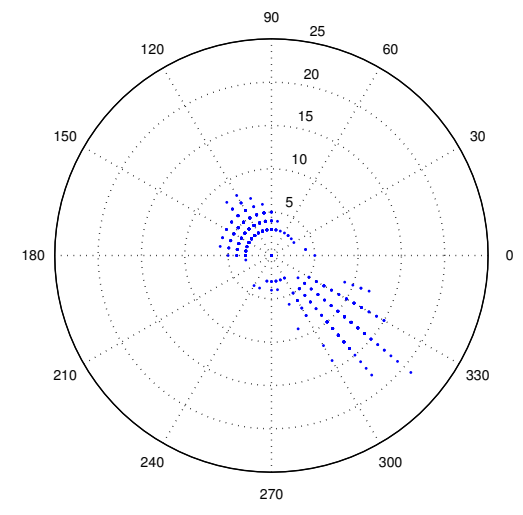

Figure 1: The Wind data Lattice. The polar plot is the representation the wind speed (in knots) and direction (in degrees) as a single complex variable [8]. The data is publicly available from the website[9].

instance a locus where the complex-valued first derivative becomes zero, or a locus where the modulus of the complex-valued signal reaches a local maximum value. In this paper a more practical definition of an extremum, namely a locus where the angle of the first derivative (first-order difference vector) changes its sign, is used. It is assumed that each local maximum is followed by a local minimum and vice versa. Note that the average of the envelopes of local maxima and minima are used, and therefore these sets can be exchanged. The envelopes can then be estimated as (complex-valued) spline interpolations of the local maxima, and minima, after which the average can be computed. The complex-valued spline is obtained by computing the spline interpolation on the real and the imaginary part separately. The rest of the procedure is same as used for the conventional EMD from Section 2. Figure 3 demonstrates the results of the interpolation and extrema detection algorithm. Notice that the extrema have been correctly identified and the mean of the envelopes correctly follows behaviour changes in the signal.

The advantage of this technique over the technique proposed in [5] is that signal does not have to be split into two parts. It also has the potential to be extended naturally to higher dimensional cases as the definition of extrema, in general, does not depend on the dimension of the data and the extension of the interpolation technique with higher-dimension splines is well understood. The problem of different type and number of IMFs for the positive and negative frequency component of the same signal and the ambiguity at zero frequency does not arise due to the unified approach adopted.

\section{SIMULATIONS AND DISCUSSIONS}

Following the analysis in [5], this Section provides the results of application of the proposed technique to the wind data shown in Figure 1. Since the IMFs are complex and they are not analytic, Figures 4 and 5 show respectively the magnitude and the phase of the IMFs. Observe that as the extrema are found based on the derivative of phase of the signal, the variations in the phase are decreasing from IMFs $c_{1}$ to $c_{9}$ (Figure 5). Since we are not calculating these extrema on the basis of absolute value of phase, this decomposition is 'rotation invariant'. This property is important for the analysis of the modality(gust, breeze, microburst) and future prediction of wind data. Although, the time-scales for the magnitude of IMFs are also increasing as we go from IMFs $c_{1}$ to $c_{9}$ in Figure 4 however this can not be guaranteed since the magnitude of the data is not used as the criterion for identification of the extrema. Despite its practical
EMD of a Complex Wind Signal-IMFs for $x_{+}[n]$

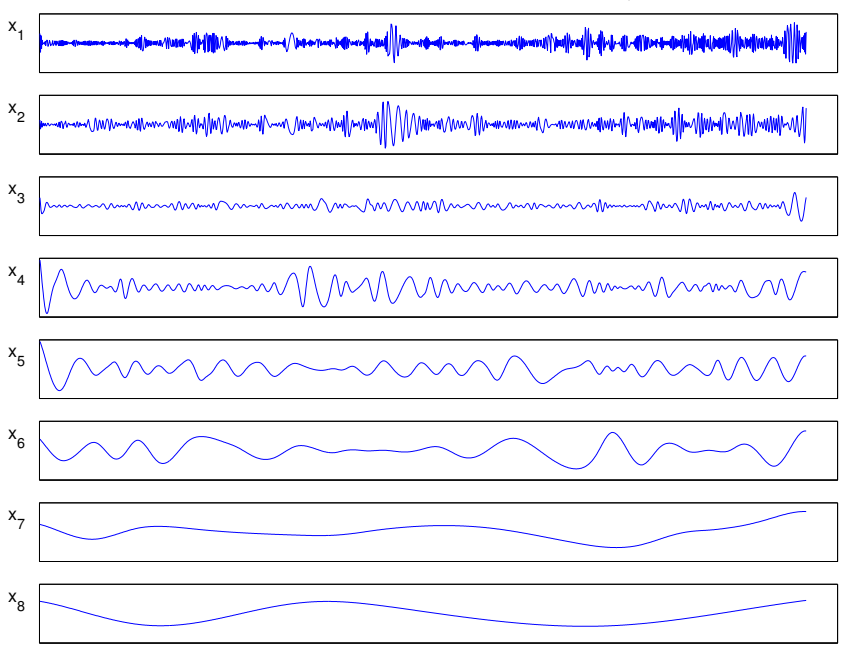

(a) IMFs for the positive frequency part

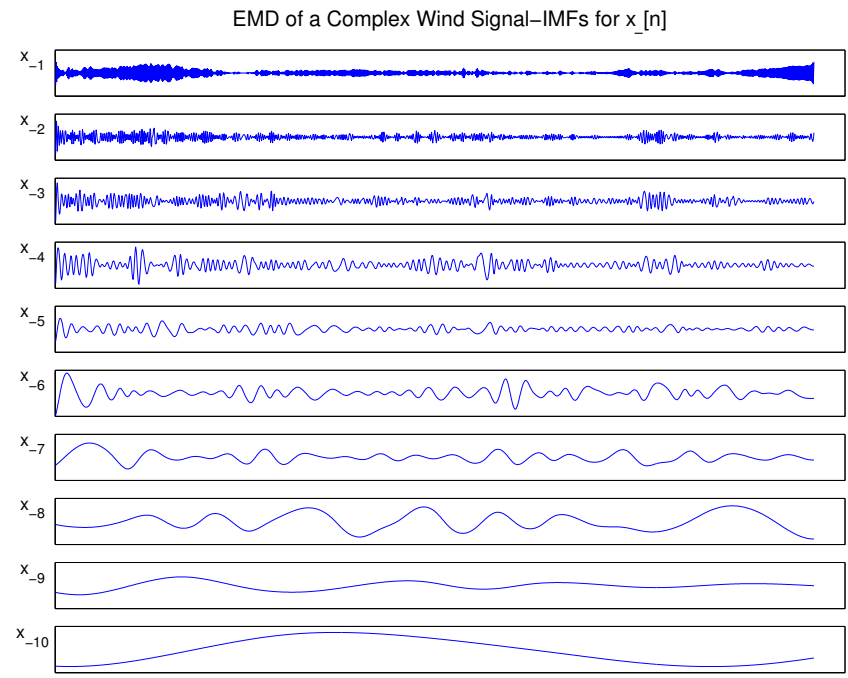

(b) IMFs for the negative frequency part

Figure 2: IMFs for the complex EMD of wind data [5]

usefulness, the derivative of the phase might not be able to identify some extrema, even from a graphical point of view (Figure 3).

The proposed approach enables us to have the same number of IMFs for the amplitude and phase, together with IMFs having physical interpretations. The polar plot of the IMFs (In Figures 6a and 6b) illustrates the regularity of IMFs, already seen in Figure 5, but with much more insight. This way IMFs generated using the proposed rotation invariant complex EMD can reflect the level of detail at different time-scales, also giving physical meaning to IMFs (trend, noise, dominant directions).

\section{CONCLUSION}

We have introduced a new Complex Empirical Mode Decomposition (EMD) method, which is a generalization of the real-valued EMD. This technique is more generic, unlike previous methods of the kind since it operates completely in $\mathbb{C}$. The analysis of real- 


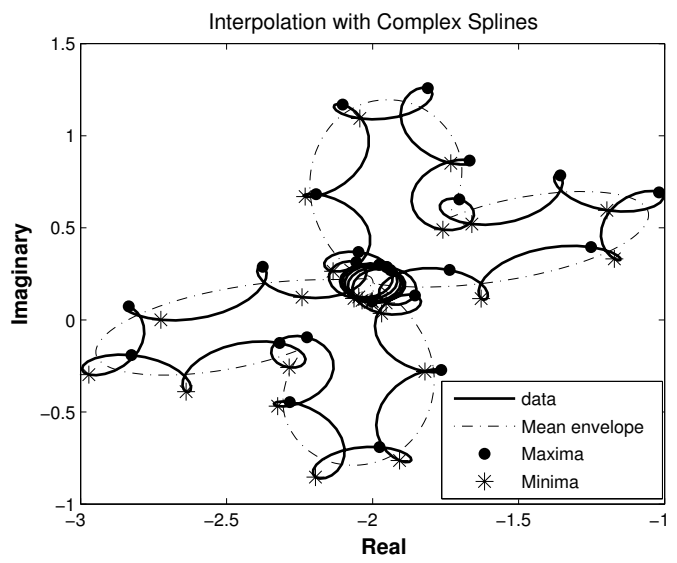

Figure 3: The extrema and mean envelope for a complex signal using the proposed complex EMD.

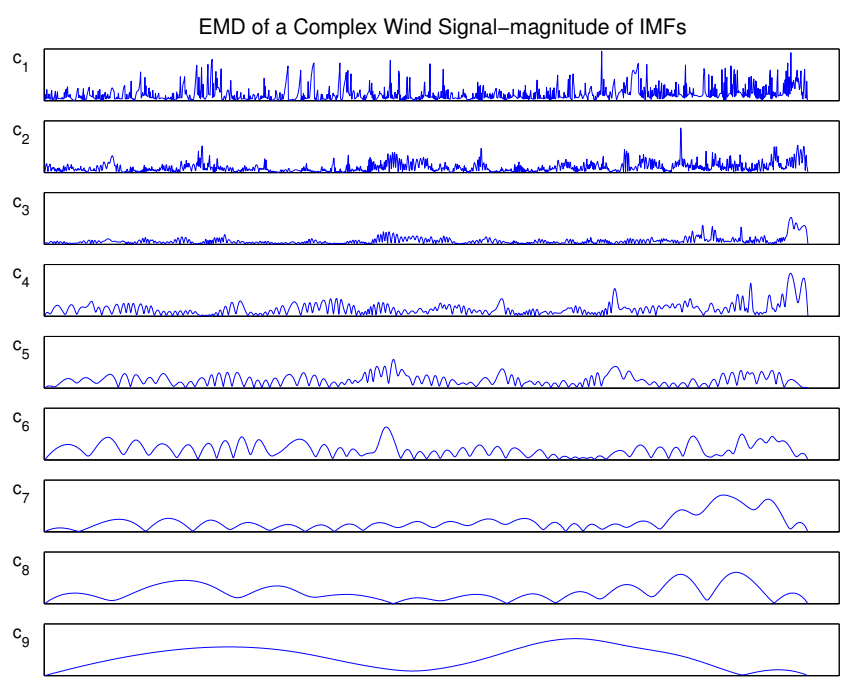

Figure 4: Proposed Method: The plot of $\|I M F s\|$

world complex-valued wind data shows that the proposed method provides new insights into the time-frequency analysis of non-linear and non-stationary signals which has not been possible with the previous methods.

\section{REFERENCES}

[1] N. Huang, Z. Shen, S. Long, M. Wu, H. Shih, Q. Zheng, N. Yen, C. Tung, and H. Liu, "The empirical mode decomposition and hilbert spectrum for nonlinear and nonstationary time series analysis," Proc. Roy. Soc. London A, vol. 454, pp. 903-995, 1998.

[2] K. T. Coughlin and K. K. Tung, "11-year solar cycle in the stratosphere extracted by the empirical mode decomposition method," Advances in Space Research, vol. 34, no. 2, pp. 323329, 2004.

[3] D. J. Duffy, "The application of Hilbert-Huang transforms to meteorological datasets," Journal of Atmospheric and Oceanic Technology, vol. 21, no. 4, pp. 599-611, 2004.
EMD of a Complex Wind Signal-Phase of IMFs

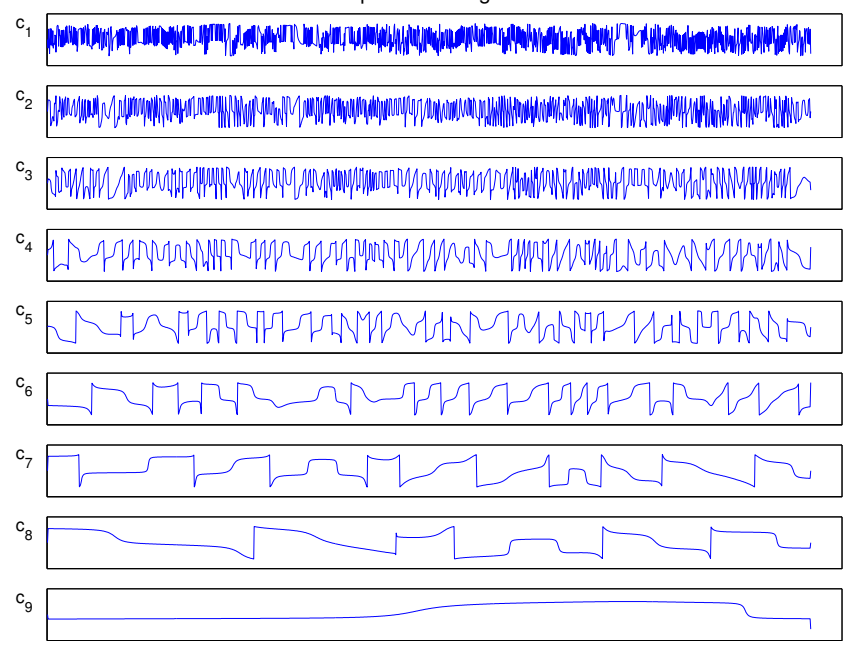

Figure 5: Proposed Method: The plot of $\angle I M F s$

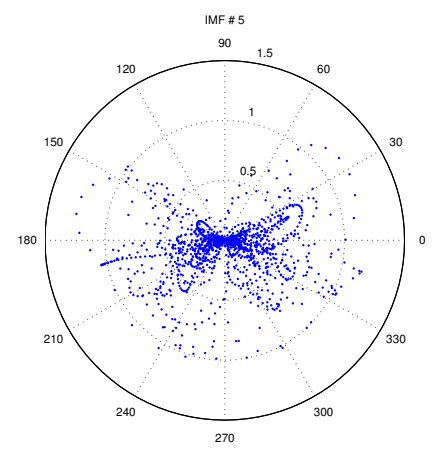

(a) IMF \# 5

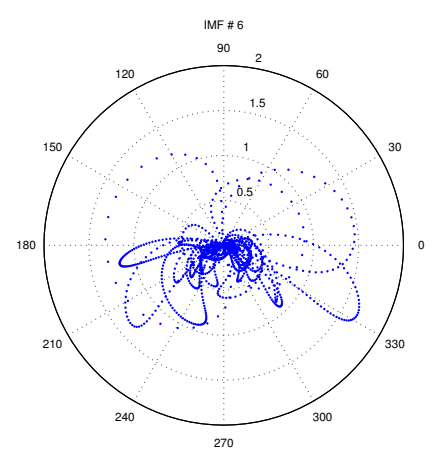

(b) IMF \# 6
Figure 6: Proposed Method: The polar plot for the IMFs

[4] S. R. Long, "Applications of HHT in image analysis," in HilbertHuang Transforms and Its Applications (N. E. Huang and S. S. P. Shen, eds.), vol. 5, ch. 13, pp. 289-305, World Scientific Publishing, 2005.

[5] T. Tanaka and D. P. Mandic, "Complex empirical mode decomposition." Accepted for IEEE Signal Processing Letters, June 2006.

[6] P. Flandrin, G. Rilling, and P. Gonçalvés, "Empirical mode decomposition as a filter bank," IEEE Signal Processing Letters, vol. 11, no. 2, pp. 112-114, 2004.

[7] S. L. Hahn, Hilbert Transforms in Signal Processing. Artech House, 1995.

[8] S. L. Goh, M. Chen, D. Popovic, D. Obradovic, K. Aihara, and D. P. Mandic, "Complex-valued forecasting of wind profile," Renewable Energy, vol. 31, pp. 1733-1750, 2006.

[9] "IEM-AWOS 1 minute data download." http://mesonet.agron.iastate.edu/request/awos/1min.php, August 2001-2006. Iowa State University Department of Agronomy. 